\title{
Canal wall reconstruction and mastoid obliteration with composite multi-fractured osteoperiosteal flap
}

\author{
Cevat Uçar
}

Received: 3 March 2006/ Accepted: 18 August 2006/Published online: 28 September 2006

(C) Springer-Verlag 2006

\begin{abstract}
We used inferior pedicled composite multifractured osteoperiosteal flap (CMOF), our original and new surgical approach, to obliterate the mastoid cavity and reconstruct the external auditory canal (EAC) to prevent the open cavity problems. CMOF was used to obliterate the mastoid cavity and reconstruct the EAC in 24 patients ( 13 women, 11 men; age span 12-51 years) who underwent radical mastoidectomy to treat the chronic otitis media between 1998 and 2004. Small meatoplasty was done in all 24 patients to relive their aesthetical concerns. Temporal bone CT scanning was done to observe the neo-osteogenesis in the mastoidectomy cavity and the CMOF, and the EAC volume was measured postoperatively. All our patients were followed-up for 2 years. The epithelization of the new EAC in our patients was complete at the end of the second month. Cholesteatoma, granulation, and recurrence of osteitis did not occur in any of the patients. We saw the new bone formation filling the mastoid cavity in the postoperative temporal bone CT scanning images. The mean volume of the new EAC on the 24th month was $1.83 \pm 0.56 \mathrm{~cm}^{3}$. We had an almost natural EAC, which owed its existence to the
\end{abstract}

This technique, composite multi-fractured osteoperiosteal flap, was presented in the symposium of Gulhane Military Medical Academy on otology and audiology, 15-18 September, 2005, Antalya, Turkey.

C. Uçar $(\bowtie)$

ENT Department, Private Türkiye Hospital, Darülaceze cad, Nadide sok. no. 7, Şişli, 80270 Istanbul, Turkey

e-mail: hcucar@yahoo.com neo-osteogenesis that grows behind the CMOF, which we use to obliterate the mastoid cavity and to reconstruct the EAC.

Keywords Mastoid obliteration - Radical mastoidectomy - Canal wall reconstruction . Osteoperiosteal flap $\cdot$ Neo-osteogenesis

\section{Introduction}

Open cavity mastoidectomy techniques applied for chronic otitis media cause some cavity problems. These patients need life-long otologic care for the debris that accumulates due to the insufficient self-cleaning mechanism of mastoidectomy cavities. Sixteen percent of ears without cavities needs cleaning, whereas this ratio climbs to $42 \%$ for ears with cavities [11]. These patients complain of dizziness in cold weather and during swimming. Other problems are non-aesthetic meatoplasty and difficulties in the placement of hearing-aid devices $[2,7]$.

Various obliteration and external auditory canal (EAC) reconstruction techniques have been recommended to eliminate open cavity problems. Mosher was the first to use soft tissue flap in 1911 and has described the postaural subcutaneous flap technique for cavity obliteration [10]. In the following years soft tissue flaps, free bone, cartilage grafts, autograft and synthetic filling substances have been used to reduce mastoid cavities [1]. Mastoid cavity obliteration by neo-osteogenesis under the periosteal flap has been first shown by Kahramanyol in 1992 [8]. This result has been my start-point and I have used inferior pedicled CMOF, which is our modification for EAC 
reconstruction and to reduce the volume of radical mastoidectomy cavity. In addition, small meatoplasty was done to lessen aesthetic concerns.

\section{Materials and methods}

Twenty-four patients (13 women and 11 men ranging in age from 12 to 51 years) were operated between 1998 and 2004. Various stages of cholesteatoma were detected during preoperative otoscopy and intraoperative observations in all those patients who had otorrhea and hearing loss for years. Four patients had complications such as facial paralysis, labyrinthine fistula and intracranial abscess. Ossicle chain injury of various severities was present in all these participants.

All patients were operated under general anaesthesia. Prior to operation, each patient had otoscopic examination, pure tone and speech audiometry and temporal bone CT scans. Approval for radical mastoidectomy indication was granted after simple mastoidectomy and atticotomy.

\section{Operative technique}

The incision line that was $1.5-2 \mathrm{~cm}$ posterior to the postauricular sulcus was kept as a margin as the subcutaneous tissues were dissected into fascia. Dissection was advanced to the skin of the posterior meatal wall along the fascia. The skin of the posterior meatal wall was horizontally transected between 6 and 12 O' clock. Then, the periosteum was incised till the bone was reached about $1-2 \mathrm{~mm}$ anteriorly to the postauricular incision. The superior part of the incision runs parallel to the inferior border of the temporalis muscle. From the upper margin of the incision that extends to the bone, the periosteum was elevated to the inferior pedicle with a 7-mm chisel. During this procedure bone lamellae with utmost $1-\mathrm{mm}$ thickness were raised as a part of a fractured bone panel attached to the periosteum. By this way the mastoid apex was reached inferiorly and EAC skin posteriorly. Periosteum was dissected from the EAC posterior wall skin. Thus, $\mathrm{CMOF}$ with an inferior base was formed. The bone lamellae under the periosteum were fractured into fragments as much as possible (Fig. 1). The flap was given the functionality of a flexible metal watch belt to place it properly into the mastoid cavity and let it reconstruct the EAC. The flap was retracted inferiorly to be able to work on the mastoid cortex.

The following rules were attended during radical mastoidectomy. (1) All bony buttresses were excised and lowered as much as possible. This is called sauc-

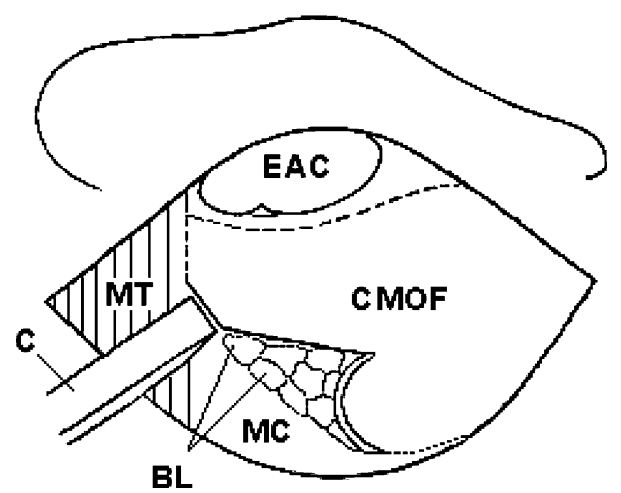

Fig. 1 Preparation of the composite multi-fractured osteoperiosteal flap; $C M O F$ composite multi-fractured osteoperiosteal flap, $M T$ musculus temporalis, $B L$ bone lamellae, $E A C$ external auditory canal, $M C$ mastoid cortex, $C$ chisel

erization or skeletonization. (2) The mastoid apex was excised. (3) All related anatomic structures and loci were visualized. (4) All diseased tissues were removed. (5) White cortical bony tissues were reached in all directions. Moreover, large defects at the dural plate and or the sigmoid sinus were reconstructed with modelled autologous bone chips obtained from the operative field.

Cleansing of the tympanic cavity was emphasized. Patients with bone conduction thresholds better than $35 \mathrm{~dB}$ and an open eustachian ostium and a healthy peri-eustachian mucosa were treated with tympanic reconstruction. The prepared CMOF was placed over the facial ridge and attached to the anterior attic wall in all cases. The flap could be elongated to the attic with the help of small incisions. The bone lamellae beneath the periosteum are supportive material to our flap and thus became a skeleton for the expected EAC. Absorbable gelatine sponge was laid over the mastoid bone behind the CMOF. The EAC skin was incised longitudinally at the $6 \mathrm{O}^{\prime}$ clock direction and the resultant skin flap having its nourishing root at the zygoma was laid posteriorly and inferiorly over the CMOF. We cared to keep the skin margins over the fascial flap. That is how the new EAC was composed. Then EAC was filled with absorbable gelatine sponge (Fig. 2). Siebenmann technique was used to form a small meatoplasty. A "Y" incision was used with its concavity looking to the posterior to elevate a flap of choncal skin and a sufficient part of the choncal cartilage was excised. The triangular skin flaps were just laid down such that they could touch the CMOF. The EAC in expectation was filled with absorbable gelatine sponge and extraphore with antibiotic ointment. The subcutaneous and cutaneous postauricular incisions were closed (Fig. 3). 

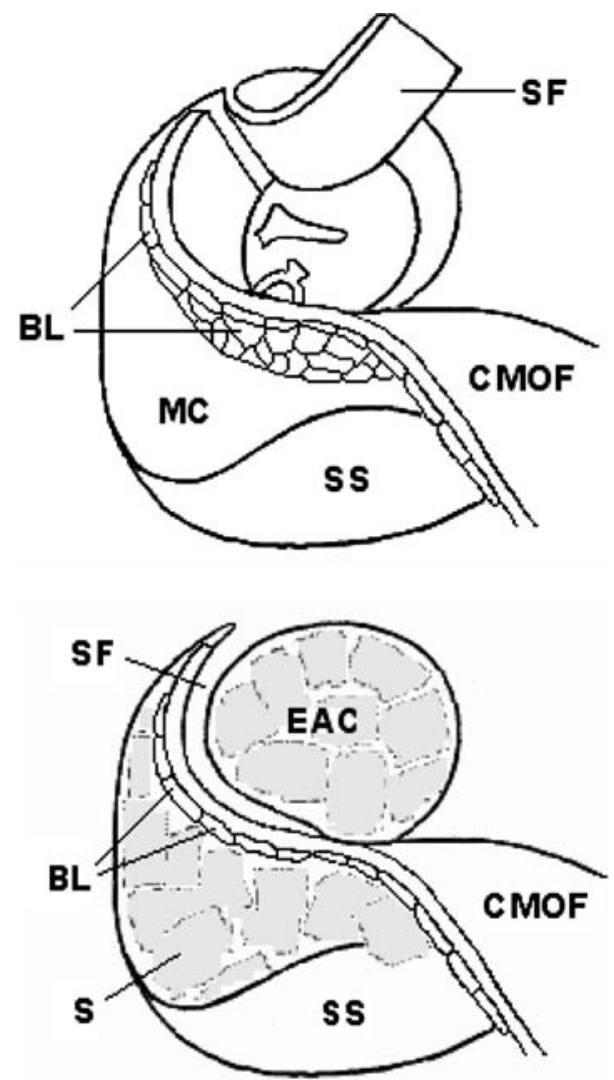

Fig. 2 Transposition of the composite multi-fractured osteoperiosteal flap (above), and fixation of it (below); $S F$ skin flap, $M C$ mastoid cavity, $C M O F$ composite multi-fractured osteoperiosteal flap, $S S$ sigmoid sinus, $B L$ bone lamellae, $E A C$ external auditory canal, $S$ sponge

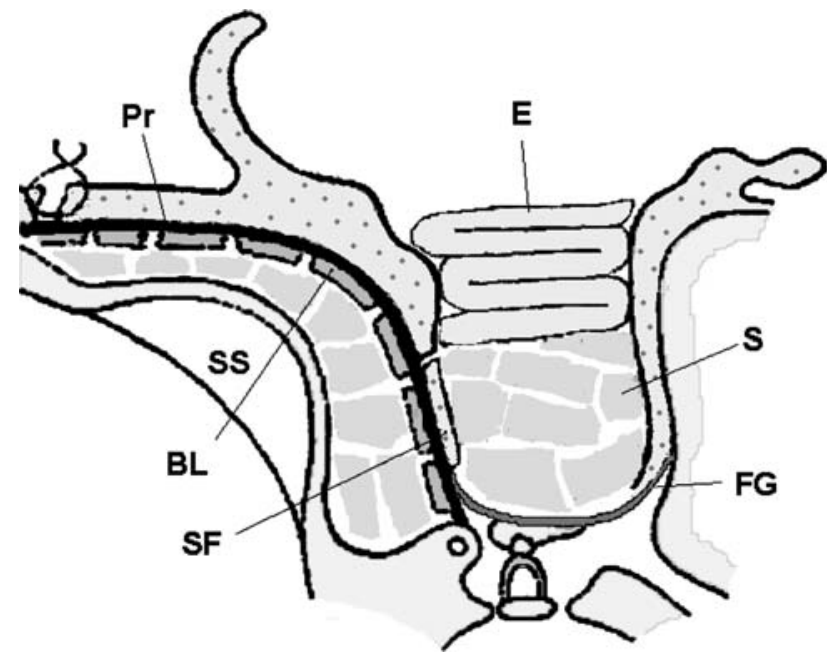

Fig. 3 Shematic represantation of surgical procedure. $\operatorname{Pr}$ Periosteum, $B L$ bone lamella, $E$ extraphore, $S$ sponge, $F G$ fascia graft, $S F$ skin flap, $S S$ sigmoid sinus

Postoperative antibiotics and anti-inflammatory medicines were continued for 1 week. Skin sutures were removed on the 7 th postoperative day. The cavity dressings were removed on the 14th day. Antibiotic and steroid drops were used at the end of the second postoperative month. In all patients epithelization of the cavity completed at the end of the 2 nd month.

Temporal bone CT scanning was done to observe the neo-osteogenesis in the mastoidectomy cavity and the CMOF on the 1st, 12th and 24th months. The EAC volume was measured on the second, 6th, 12th and 24th months. The volume measurements were done with saline, warmed up to the body temperature that was filled into the EAC up to the level of the posterior line of the meatoplasty. The head position was horizontal during the measurement. Data of the study were analysed by the statistical package for social sciences (SPSS) for Windows 10.0.

\section{Results}

This study was done between the years 1998 and 2004 in 24 patients. All patients were followed-up for at least 2 years. EACs were smooth and healthy. In all patients epithelization was complete after the 2nd month of the operation. None had recurrent osteitis, cholesteatoma or granulation. Otoscopic examination could reveal the entire cavity in all these cases and debris and cerumen cleaning was feasible.

The mean volume of the new EAC on the 2nd, 6th, 12 th and 24th months were $0.93 \pm 0.32,1.43 \pm 0.48$, $1.66 \pm 0.53,1.83 \pm 0.56 \mathrm{~cm}^{3}$, respectively. The canal volume measurements of the patients that we were able to follow after 24 months revealed no significant difference. The evaluations of the postoperative temporal bone CT scanning revealed no retraction or displacement around, but demonstrated new bone formation obliterating the mastoid cavity behind the CMOF that was used to build the posterior wall of the EAC (Fig. 4). The CMOF never displaced toward the EAC or the sigmoid sinus, and it always remained where it was placed during the operation.

None of our patients returned with vertigo induced by cold air or swimming. No aesthetical disapproval occurred. Introducing the hearing aid placements into the EAC was easy and almost natural. The therapeutical results in four patients with preoperative complications were successful. In one patient conchal perichondritis emerged soon after the operation and was treated with medical therapy.

\section{Discussion}

Various treatments have been proposed for mastoid cavity obliteration and reconstruction $[1,10]$. Extended 
Fig. 4 Postoperative

temporal bone CT images

1 month later (a), and 2 years later (b)
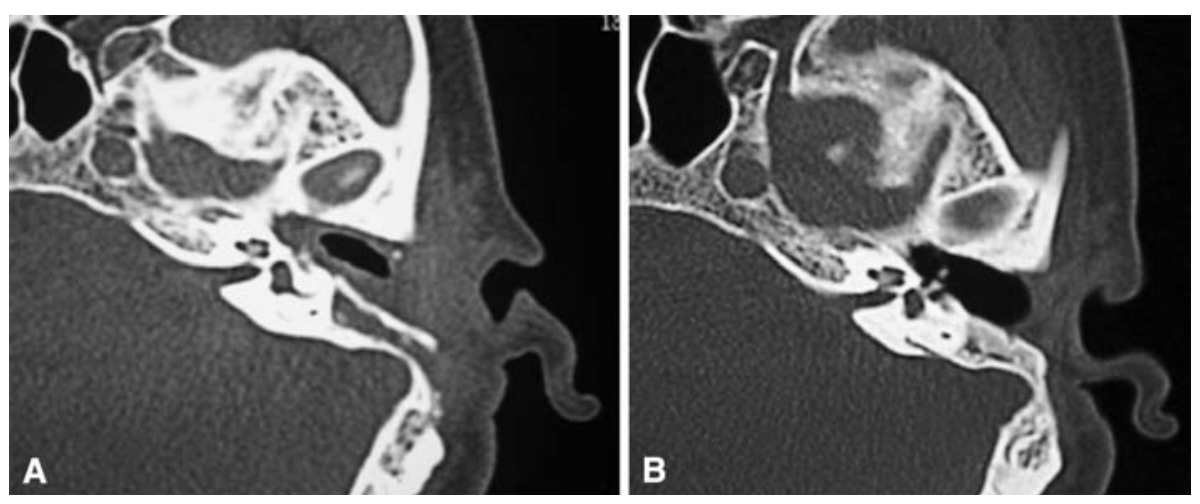

meatoplasty has been used for auto-cleansing and sufficient aeration $[4,8,13]$. Some authors offered a small meatoplasty to avoid the inevitable aesthetic concerns stemming from a wider meatoplasty [6]. However, small meatoplasty has restricted the cavity drainage and aeration by increasing the cavity depth posteriorly. In our technique a small meatoplasty became a functional continium of the almost a normal EAC anatomy that was obtained by the placement of the CMOF to the posterior edge of the meatoplasty. This newly formed EAC has provided sufficient drainage and aeration, and a preferable aesthetic appearance (Fig. 5).

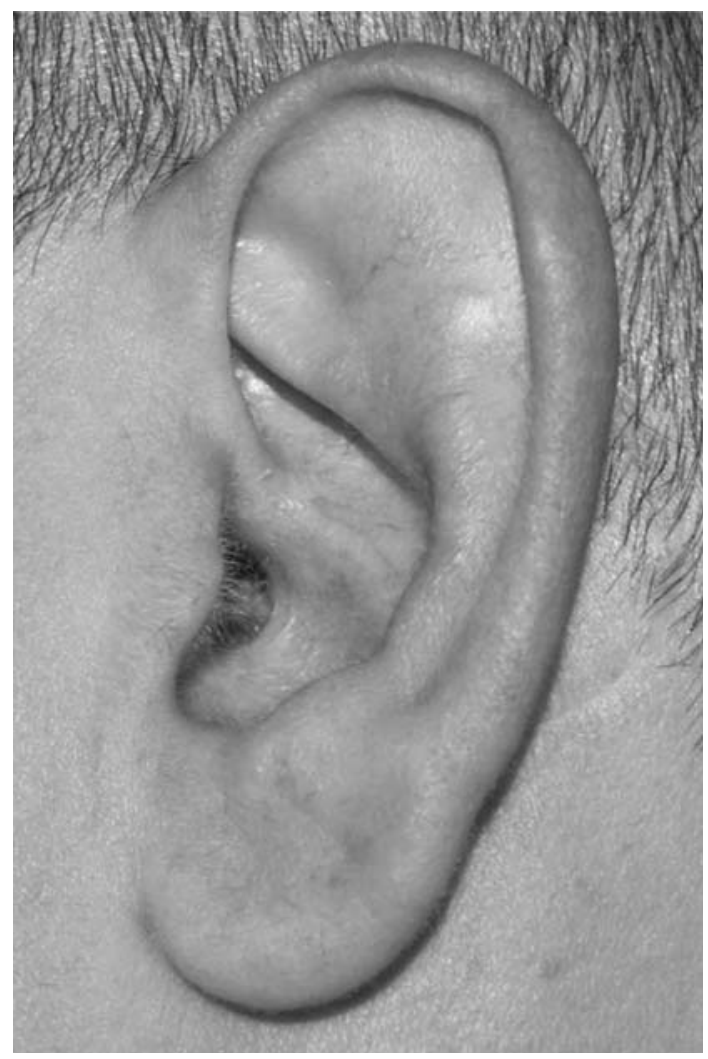

Fig. 5 The external ear meatus in the second postoperative year
The bilateral perfusion obtained by the postauricular artery supplying the inferior pedicled CMOF and the branches of internal maxillary artery supplying the superior pedicled EAC flap have accelerated cavity healing and epithelization. The absorbable gelatine sponges placed into the mastoidectomy cavity and new EAC have helped the immobilization of the CMOF by supporting it placed at the facial ridge. Moreover, they have contributed to the formation of a homogenous haematoma under the CMOF. Many authors have detected that haematoma at the fracture site has aided in fracture healing [5, 12]. Numerous callus areas emerging in interlamellar fracture lines under the CMOF have supplied both skeletal support and obliteration of the mastoidectomy cavity by neo-osteogenesis.

Histological investigations relating fracture-healing process have shown that intramembranous bone formation begins under the periosteum in a few days after fracture formation and bridging between fracture fragments begins in 4 weeks [3]. We observed calcification foci indicating osteoblastic activity in histological investigations of a cavity wall biopsy taken from a patient in 6 months after surgery (Fig. 6). New bone formation filling the mastoidectomy cavity under the CMOF was detected in the postoperative temporal bone CT images taken in the 1st, 12th and 24th months.

In a study where Palva flap was used for mastoid obliteration, the ear canal was found enlarged at both the early and the late postoperative check-up compared to the preoperative values [11]. The results of the studies, reported by Kahramanyol et al. [8] in which mastoid cavities were obliterated with fascioperiosteal flap, have shown that the mastoid cavity volume was decreased by $39 \%$ due to neo-osteogenesis under fascioperiosteal flap, and that the obliteration has stayed in long term has shown radiologically [9]. In our study it has shown a nearly twofold increase in EAC volume and no significant difference after 24th months. Thus the difference in EAC volumes between the 2nd month 


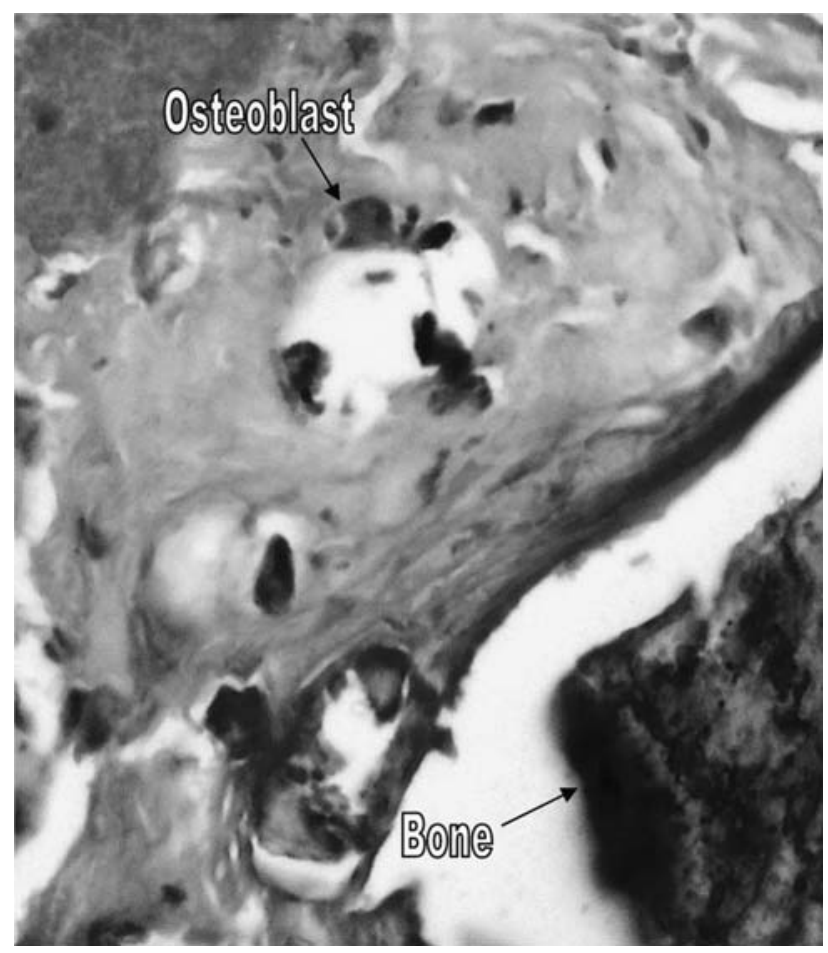

Fig. 6 Calcification foci indicating osteblastic activity are seen in histologic investigations 6 months after surgery $(\mathrm{HE} \times 400)$

and the 2nd year of the postoperative period was assessed as a result of shrinking of the tissues, composing the EAC, especially the periosteum.

\section{Conclusion}

Some large meatoplasties performed for open cavity problems are not preferable due to non-aesthetic appearance. On the other hand, small meatoplasties that are preferred to overcome this concern cause aeration and drainage problems by increasing the depth of mastoidectomy cavity posteriorly. Therefore, in this study we both performed small meatoplasties to avoid the aesthetic concerns and at the same time filled the mastoid cavity up to the posterior edge of the meatoplasty to obtain efficient aeration and drainage.

\section{References}

1. Black B (1998) Mastoidectomy elimination. Am J Otol 19:551-557

2. Dornhoffer JL (1999) Surgical modification of the difficult mastoid cavity. Otolaryngol Head Neck Surg 120:361-367

3. Einhorn TA (1998) The cell and molecular biology of fracture healing. Clin Orthop Relat Res 355(Suppl):S7-21

4. Görür K, Özcan C, Ünal M, Vayısoğlu Y (2002) Causes of failure in open cavity mastoidectomy. KBB İhtisas Dergisi 9:179-183

5. Grundnes O, Reikeras O (1993) The importance of the hematoma for fracture healing in rats. Acta Orthop Scand 64:340-342

6. Hoffmann DF, Fagan PA, Taylor B (1997) Reduction meatoplasty. Laryngoscope 107:1127-1128

7. Jackson CG, Schall DG, Glasscock ME 3rd, Macias JD, Widick MH, Touma BJ (1996) A surgical solution for the difficult chronic ear. Am J Otol 17:7-14

8. Kahramanyol M (1992) Fascioperiosteal flap and neoosteogenesis in radical mastoidectomy. Ear Nose Throat J 71:7077

9. Kahramanyol M, Özünlü A, Pabuşçu Y (2000) Fascioperiosteal flap and neo-osteogenesis in radical mastoidectomy: long-term results. Ear Nose Throat J 79:524-526

10. Mosher HP (1911) A method of filling the excavated mastoid with a flap from the back of the auricule. Laryngoscope 21:1158-1163

11. Ojala K, Sorri M, Sipila P, Palva A (1982) Late changes in ear canal volumes after mastoid obliteration. Arch Otolaryngol 108:208-209

12. Ozaki A, Tsunado M, Kinoshita S, Saura R (2000) Role of the hematoma and periostium during fracture healing in rats: interactions of fracture hematoma and the periostium in the initial step of the healing process. J Orthop Sci 5:64-70

13. Sade J, Weinberg J, Berco E, Halvey A (1982) The marsupialized (radical) mastoid. J Laryngol Otol 96:869-875 